\title{
STIRLING NUMBER REPRESENTATION PROBLEMS
}

\author{
H. W. GOULD
}

1. Introduction. The Stirling numbers of the first kind are defined as the coefficients $S_{1}(n, k)$ in the expansion

$$
\prod_{k=0}^{n}(1+k x)=\sum_{k=0}^{\infty} S_{1}(n, k) x^{k},
$$

so that [6] $S_{1}(n, k)=$ the sum of the $C_{n, k}$ possible products, each with $k$ different factors, which may be formed from the first $n$ natural numbers.

The Stirling numbers of the second kind are defined as the coefficients $S_{2}(n, k)$ in the expansion

$$
\prod_{k=0}^{n}(1-k x)^{-1}=\sum_{k=0}^{\infty} S_{2}(n, k) x^{k},
$$

so that $S_{2}(n, k)=$ the sum of the $C_{(n+k-1), k}$ possible products, each with $k$ factors (repetition allowed), which may be formed from the first $n$ natural numbers.

Schlömilch [9] found the formula

$$
\begin{aligned}
& (-1)^{k} S_{1}(n-1, k) \\
& =(n-k)\left(\begin{array}{l}
n \\
k
\end{array}\right)\left(\begin{array}{c}
n+k \\
k
\end{array}\right) \sum_{j=0}^{k}(-1)^{i}\left(\begin{array}{l}
k \\
j
\end{array}\right) \frac{S_{2}(j, k)}{(n+j)\left(\begin{array}{c}
k+j \\
j
\end{array}\right)},
\end{aligned}
$$

which is one of the simplest known explicit representations of the Stirling numbers of the first kind in terms of the Stirling numbers of the second kind. By means of a simple binomial coefficient identity this formula is seen to be equivalent to the neater formula

$$
S_{1}(n-1, k)=\sum_{j=0}^{k}\left(\begin{array}{c}
k+n \\
k-j
\end{array}\right)\left(\begin{array}{c}
k-n \\
k+j
\end{array}\right) S_{2}(j, k),
$$

found by L. Schläfli [8].

These two formulas do not seem to be very well known, perhaps because it is easier to calculate $S_{1}$ by means of recurrence formulas.

Of course, it is well known $[4 ; 5]$ that $S_{2}$ is given by the very simple formula

Received by the editors June 25, 1959 and, in revised form, August 14, 1959. 


$$
\begin{aligned}
S_{2}(n, k) & =\left.\frac{1}{n !} \Delta_{x, 1}^{n} x^{n+k}\right|_{x=0} \\
& =\frac{(-1)^{n}}{n !} \sum_{j=0}^{n}(-1)^{j}\left(\begin{array}{l}
n \\
j
\end{array}\right) j^{n+k} .
\end{aligned}
$$

We remark that the numbers $S_{2}$ occur in the familiar Newton-Gregory expansion $[5 ; 12]$ of $x^{n}$ :

$$
x^{n}=\sum_{k=0}^{n} k ! S_{2}(k, n-k)\left(\begin{array}{l}
x \\
k
\end{array}\right) .
$$

In this paper we offer simple proofs of the following formulas:

$$
(-1)^{k} S_{1}(n-1, k)=\left(\begin{array}{c}
n-1 \\
k
\end{array}\right) \sum_{j=0}^{k}(-1)^{j}\left(\begin{array}{c}
k+1 \\
j+1
\end{array}\right) \frac{S_{2}(j n, k)}{\left(\begin{array}{c}
k+j n \\
k
\end{array}\right)}
$$

$$
(-1)^{k} S_{2}(n, k)=\left(\begin{array}{c}
k+n \\
k
\end{array}\right) \sum_{j=0}^{k}(-1)^{j}\left(\begin{array}{c}
k+1 \\
j+1
\end{array}\right) \frac{S_{1}(j n-1, k)}{\left(\begin{array}{c}
j n-1 \\
k
\end{array}\right)},
$$

$$
S_{2}(n-k, k)=\sum_{j=0}^{k}\left(\begin{array}{c}
k-n \\
k+j
\end{array}\right)\left(\begin{array}{c}
k+n \\
k-j
\end{array}\right) S_{1}(k+j-1, k),
$$

$$
S_{1}(n-1, k)=\sum_{t=0}^{k} K(t) S_{1}(k+t-1, k),
$$

$$
S_{2}(n-k, k)=\sum_{t=0}^{k} K(t) S_{2}(t, k),
$$

where in (1.10) and (1.11)

$$
K(t)=\sum_{j=0}^{k}\left(\begin{array}{c}
k-n \\
k+j
\end{array}\right)\left(\begin{array}{c}
k+n \\
k-j
\end{array}\right)\left(\begin{array}{c}
2 k+j \\
k-t
\end{array}\right)\left(\begin{array}{c}
-j \\
k+t
\end{array}\right) .
$$

In particular we remark that (1.9) is a companion to (1.4) thereby providing a simple way to express the Stirling numbers of the second kind explicitly in terms of the Stirling numbers of the first kind.

We also make application of the Eulerian numbers $[1 ; 12]$

$$
A_{n, k}=\sum_{j=0}^{k}(-1)^{i}\left(\begin{array}{c}
n+1 \\
j
\end{array}\right)(k-j)^{n},
$$

in order to show that 


$$
\begin{aligned}
S_{2}(n-k, k)= & \frac{(-1)^{k}}{n !}\left(\begin{array}{l}
n \\
k
\end{array}\right) \sum_{i=0}^{k}(-1)^{i} S_{1}(k-1, k-i) \\
& \cdot \sum_{j=0}^{n} A_{n, j}(j-1)^{i} .
\end{aligned}
$$

2. Proof of (1.7) and (1.8). Because of the relations

(2.1) $\left(\begin{array}{c}n-1 \\ k\end{array}\right) B_{k}^{(n)}=(-1)^{k} S_{1}(n-1, k), \quad n$ positive integer,

and

$$
\left(\begin{array}{c}
n+k \\
k
\end{array}\right) B_{k}^{(-n)}=S_{2}(n, k), \quad n \text { positive integer, }
$$

where $B_{\mathfrak{k}}^{(x)}=B_{\mathfrak{k}}^{(x)}(0)$ is a generalized Bernoulli number and [7]

$$
\left(\frac{z}{e^{z}-1}\right)^{x} \cdot e^{t z}=\sum_{k=0}^{\infty} B_{k}^{(x)}(t) \frac{z^{k}}{k !}
$$

and also in view of the relations [3]

$$
\begin{aligned}
& S_{1}(-n-1, k)=S_{2}(n, k), \\
& S_{2}(-n-1, k)=S_{1}(n, k),
\end{aligned}
$$

it will be sufficient to establish for all real $n$ that

$$
B_{k}^{(n)}=\sum_{j=0}^{k}(-1)^{j}\left(\begin{array}{c}
k+1 \\
j+1
\end{array}\right) B_{k}^{(-j n)},
$$

and then (1.7) and (1.8) are special cases.

We take the generalized chain rule of differentiation in the form (cf. $[5$, p. 216] and $[6$, p. 22] in general)

$$
D_{x}^{k}\left(\frac{1}{z}\right)=\sum_{j=0}^{k}(-1)^{j}\left(\begin{array}{c}
k+1 \\
j+1
\end{array}\right) \frac{1}{z^{j+1}} D_{x^{z}}^{k j}
$$

and define

$$
z=\left(\frac{e^{x}-1}{x}\right)^{n}
$$

Then noting that $\lim _{x \rightarrow 0} z=1$, and that $[4 ; 7]$

$$
B_{k}^{(n)}=\left.D_{x}^{k}\left(\frac{1}{z !}\right)\right|_{x=0},
$$


we find that (2.5) follows immediately from (2.6).

3. Proof of (1.9). We have [7, p. 147]

$$
\left(\frac{\log (x+1)}{x}\right)^{n}=n \cdot \sum_{k=0}^{\infty} \frac{x^{k}}{k !} \frac{B_{k}^{(n+k)}}{n+k}, \quad|x|<1 .
$$

From this and $[7$, p. 145$]$

$$
B_{k}^{(n+1)}(1)=\left(1-\frac{k}{n}\right) B_{k}^{(n)},
$$

it follows that

$$
\left(\frac{x}{\log (x+1)}\right)^{n}=\sum_{k=0}^{\infty} \frac{x^{k}}{k !} B_{k}^{(k-n+1)}(1) .
$$

Now the generalized chain rule may also be written in the convenient form (cf. $[5$, p. 216] and $[6$, p. 22])

$$
z^{n} D_{x}^{k} z^{-n}=\sum_{j=0}^{k}\left(\begin{array}{c}
-n \\
j
\end{array}\right)\left(\begin{array}{c}
k+n \\
k-j
\end{array}\right) z^{-j} D_{x}^{k} z^{j}
$$

and by an easy binomial coefficient identity this may also be written as

$$
(-1)^{k}\left(\begin{array}{c}
n-1 \\
k
\end{array}\right) z^{n} D_{x}^{k} z^{-n}=\sum_{j=0}^{k}\left(\begin{array}{c}
k-n \\
k+j
\end{array}\right)\left(\begin{array}{c}
k+n \\
k-j
\end{array}\right)\left(\begin{array}{c}
k+j \\
j
\end{array}\right) z^{-j} D_{x}^{k} z^{j}
$$

We define

$$
z=\frac{\log (x+1)}{x}
$$

and note that $\lim _{x \rightarrow 0} z=1$. Then it follows from (3.5) and the expansions (3.1) and (3.3) together with (3.2) that

$$
(-1)^{k}\left(\begin{array}{l}
n \\
k
\end{array}\right) B_{k}^{(k-n)}=\sum_{j=0}^{k}\left(\begin{array}{l}
k-n \\
k+j
\end{array}\right)\left(\begin{array}{c}
k+n \\
k-j
\end{array}\right)\left(\begin{array}{c}
k+j-1 \\
k
\end{array}\right) B_{k}^{(j+k)}
$$

and consequently when we apply (2.2) to the left-hand member and (2.1) to the right-hand member, this expression becomes identically (1.9) which is therefore proved.

4. Proof of (1.10) and (1.11). It is a routine calculation to substitute for $S_{2}(j, k)$ in (1.4) by means of (1.9) and obtain (1.10). Likewise we substitute for $S_{1}(k+j-1, k)$ in (1.9) by means of (1.4) and the result is exactly (1.11). The summation $K(t)$ occurs in each case. 
5. Proof of (1.14). Worpitzky [12, formula (14)] has shown that

$$
k ! S_{2}(k, n-k)=\sum_{j=0}^{n}\left(\begin{array}{c}
j-1 \\
n-k
\end{array}\right) A_{n, j},
$$

where $A_{n, j}$ are the Eulerian numbers defined by (1.13).

Now it is a consequence of (1.1) that the familiar expansion

$$
\left(\begin{array}{l}
x \\
n
\end{array}\right)=\sum_{k=0}^{n} \frac{(-1)^{n-k}}{n !} S_{1}(n-1, n-k) x^{k}
$$

is obtained.

From (5.1) we obtain, first putting $n-k$ for $k$ and then using (5.2),

$$
\begin{aligned}
(n-k) ! S_{2}(n-k, k) & =\sum_{j=0}^{n}\left(\begin{array}{c}
j-1 \\
k
\end{array}\right) A_{n, j} \\
& =\sum_{j=0}^{n} \sum_{i=0}^{k} \frac{(-1)^{k-i}}{k !} S_{1}(k-1, k-i)(j-1)^{i} A_{n, j} .
\end{aligned}
$$

Simplification of this yields relation (1.14) as proposed.

It would be interesting to obtain a relation inverse to (1.14), that is a formula expressing $S_{1}$ in terms of $S_{2}$ using $A_{n, j}$.

It is not hard to show that a relation inverse to (5.1) is

$$
(-1)^{k} A_{n, k}=\sum_{j=0}^{n}(-1)^{j}\left(\begin{array}{l}
n-j \\
n-k
\end{array}\right) j ! S_{2}(j, n-j) .
$$

\section{REFERENCES}

1. L. Carlitz, Eulerian numbers and polynomials, Math. Mag. vol. 32 (1959) pp. 247-260.

2. H. W. Gould, A theorem concerning the Bernstein polynomials, Math. Mag. vol. 31 (1958) pp. 259-264.

3. J. G. Hagen, Synopsis der höheren Mathematik, Berlin, 1891.

4. C. Jordan, On Stirling's numbers, Tôhoku Math. J. vol. 37 (1933) pp. 254-278.

5. - Calculus of finite differences, New York, 1957.

6. N. Nielsen, Handbuch der Theorie der Gammafunktion, Leipzig, 1906.

7. N. E. Nörlund, Vorlesungen über Differenzenrechnung, Berlin, 1924.

8. L. Schläfli, Ergänzung der Abhandlung über die Entwickelung des Produkts ...., J. Reine Angew. Math. vol. 67 (1867) pp. 179-182.

9. O. Schlömilch, Recherches sur les coefficients des facultés analytiques, J. Reine Angew. Math. vol. 44 (1852) pp. 344-355.

10. - Compendium der höheren Analysis, Braunschweig, 1866.

11. I. J. Schwatt, Introduction to the operations with series, University of Pennsylvania, 1924.

12. J. Worpitzky, Studien über die Bernoullischen und Eulerschen Zahlen, J. Reine Angew. Math. vol. 94 (1883) pp. 203-232.

WeST Virginia UnIVERSITY 